\title{
NONCOMMUTATIVE GEOMETRY: A QUANTUM GROUP APPROACH
}

\author{
Eliezer Batista
}

\begin{abstract}
This work is a short review on recent results about the Hopf algebraic approach to noncommutative differential geometry for a non specialist audience. This approach is different from the spectral triple formulation because it does not need an extra element, such as the Dirac operator, in order to construct a differential calculus. We show how the differential calculi on a Hopf algebra can be obtained from the structure of ideals in the Hopf algebra itself. The language of quantum groups is more appropriate to define noncommutative analogues of group actions, homogeneous spaces and principal bundles. Some geometrical properties of these noncommutative bundles are explored.
\end{abstract}

\section{Introduction}

Noncommutative geometry is a mathematical topic which grew very quickly in the past two decades. Now it takes an important part in the scientific literature both in physics and mathematics. From the physical part, noncommutative geometry opens new possibilities for model building of elementary interactions $[1,8,7]$. From the mathematical side, noncommutative geometrical methods are useful to treat classical problems in geometry, topology, probability and even number theory [6], and provides a rich source of new mathematical structures and challenging problems [10].

The origins of noncommutative geometry can be traced back to the theorem of Gelfand and Naimark, which states that every commutative $\mathrm{C}^{*}$-algebra can be characterised as an algebra of functions on a locally compact Haussdorff space. Relaxing the condition of commutativity, we can think a noncommutative $\mathrm{C}^{*}$-algebra as a "noncommutative space" and then work out all the 
geometric properties in a purely algebraic way. The second step towards a noncommutative geometry was done by the Serre-Swan theorem, which identifies vector bundles over a compact space with finitely generated projective modules over the algebra of functions on this space. In the 1980's, Alain Connes established the formulation of noncommutative geometry based on the concept of spectral triples. A spectral triple basically consists in a $\mathrm{C}^{*}$-algebra $A$, a representation of this algebra on a Hilbert space $\pi: A \rightarrow \mathcal{B}(H)$, and a self adjoint operator $D$ acting on $H$ such that the commutators $[D, \pi(a)]$ are bounded operators $\forall a \in A[10]$. This operator is responsible for generating the differential calculus and is named the Dirac operator because the first example of a spectral triple was exactly which describes the commutative geometry of a spin manifold. In this case, that the algebra is the commutative algebra of functions, the Hilbert space is given by the square integrable spinors and the $D$ operator is exactly the Dirac operator on that manifold. The differential calculus generated is the same as the De-Rham complex on the manifold.

At the same time, mainly in the context of integrable systems, there emerged a large class of examples of noncommutative algebras with a manifest geometrical content, the quantum groups. The quantum groups, whose underlying algebraic structure is known as Hopf algebras, appeared from two different perspectives. The first one was the deformation of Lie bialgebras [9] and the second as a noncommutative $\mathrm{C}^{*}$-algebra which deforms the relations of the coordinate algebra of a group [16]. These two approaches were shown to be dual in the sense that it is possible to identify these Hopf algebras as dual one of the other. Just as Lie groups and homogeneous spaces form a rich class of examples of manifolds in classical geometry, quantum groups provide key examples of noncommutative geometry. Notions as symmetry group and group actions can be easily translated to a noncommutative geometric context using quantum groups. The first important notion in the quantum group approach to noncommutative geometry is the concept of covariant calculus [17]. The idea is to define well behaved differential forms with respect to the symmetry given by the action of the Hopf algebra (in fact is a co-action, as we will see later). The simplest 
example is what occurs in real functions of one variable, the notion of derivative must be invariant by the action of the additive group of $\mathbb{R}$ by translations. The most important fact about covariant diferential calculi on quantum groups is that their construction depends only on the data provided by the algebra itself [12]. While in the spectral triple approach, the differential structure is generated by the "extra" element, the Dirac operator, in the quantum group approach, the calculus arises from a quotient of a universal calculus by an appropriated bimodule based upon an ideal of the algebra. Some attempts were made in the last years to make a bridge between quantum group and spectral triple approaches [5], nevertheless, there is much more to be done in order to converge these two perspectives and provide an ultimate theory.

Afterwards, in order to develop a consistent formulation of noncommutative geometry of quantum groups, there is the notion of quantum principal bundle $[3,11]$. This notion has a more abstract origin and is related with the generalization of galoisian extensions to the context of Hopf algebras, namely Hopf-Galois extensions. The existence of principal bundles in the context of quantum groups has several consequences for the study of noncommutative geometry on such spaces. The theory of connections on quantum principal bundles has interest even for physics, in investigating deformed gauge field theories [2]. Finally, the algebraic structure allows one to define associated bundles to quantum principal bundles and frames over the noncommutative manifolds leading to the construction of a noncommutative riemannian geometry.

This work is divided as follows: In section 2, we review the basics of the language of Hopf algebras, modules and co-modules. In section 3, we describe the theory of covariant differential calculi over quantum groups, giving some examples. In the last section, we develop the theory of quantum principal bundles. As this work intends to be an introduction for nonspecialist readers, some examples and proofs of theorems are worked out explicitly in order to give a didatic presentation of the concepts and techniques involved in the theory. For questions of brevity, many other interesting examples were ommited, to them I refer to the literature. 


\section{Hopf Algebraic Preliminaries}

The basic algebraic structure of quantum groups is the structure of Hopf algebra $[13,12]$.

Definition 2.1 An algebra is a complex vector space $H$ endowed with a linear map (multiplication)

$$
\begin{aligned}
\mu: H \otimes H & \rightarrow H, \\
a \otimes b & \mapsto a \cdot b
\end{aligned}
$$

which is associative, ie, $\mu \circ(\mu \otimes I d)=\mu \circ(I d \otimes \mu)$, or in terms of elements, $a \cdot(b \cdot c)=(a \cdot b) \cdot c$. Have a unit element $\mathbf{1} \in H$ such that $\mathbf{1} \cdot a=a \cdot \mathbf{1}=a$. The unit can be thought as a map

$$
\begin{aligned}
\eta: \mathbb{C} & \rightarrow H, \\
\lambda & \mapsto \lambda \mathbf{1}
\end{aligned}
$$

and its property written as $\mu \circ(\eta \otimes I d)=\mu \circ(I d \otimes \eta)=I d$. An algebra is said to be commutative if $a \cdot b=b \cdot a$.

Definition 2.2 A co-algebra $H$ is a complex vector space with two linear maps. The co-multiplication,

$$
\begin{aligned}
\Delta: H & \rightarrow H \otimes H, \\
a & \mapsto \Delta(a)
\end{aligned}
$$

which is co-associative, ie, $(\Delta \otimes I d) \circ \Delta=(I d \otimes \Delta) \circ \Delta$, and the co-unit,

$$
\begin{aligned}
\epsilon: H & \rightarrow \mathbb{C}, \\
a & \mapsto \epsilon(a)
\end{aligned}
$$

which obeys $(\epsilon \otimes I d) \circ \Delta=(I d \otimes \epsilon) \circ \Delta=I d$. A co-algebra is co-commutative if $\sigma \circ \Delta=\Delta$, where $\sigma$ denotes the flip morphism in $H \otimes H$.

There is a standard abstract notation for the co-products in terms of the elements of the algebra, namely, the Sweedler notation, written as $\Delta(a)=$ $\sum a_{(1)} \otimes a_{(2)}$. This sum is only indicative and it is well defined because the properties of the co-product. In this notation, for example, the co-unit axiom can be expressed as

$$
\sum a_{(1)} \epsilon\left(a_{(2)}\right)=\sum \epsilon\left(a_{(1)}\right) a_{(2)}=a
$$


Definition 2.3 A bi-algebra, is an algebra $H$ with co-algebra structure where both, $\Delta$ and $\epsilon$ are algebra morphisms, that is, $\Delta(a \cdot b)=\Delta(a) \cdot \Delta(b), \Delta(\mathbf{1})=\mathbf{1} \otimes \mathbf{1}$, $\epsilon(a \cdot b)=\epsilon(a) \epsilon(b)$ and $\epsilon(\mathbf{1})=1$.

Definition 2.4 A Hopf algebra is a bi-algebra $H$ endowed with a linear map,

$$
\begin{aligned}
S: H & \rightarrow H, \\
a & \mapsto S(a)
\end{aligned}
$$

called antipode satisfying $\mu \circ(S \otimes i d) \circ \Delta=\mu \circ(i d \otimes S) \circ \Delta=\eta \circ \epsilon$.

In terms of the Sweedler notation, the antipode axiom for Hopf algebras reads

$$
\sum a_{(1)} S\left(a_{(2)}\right)=\sum S\left(a_{(1)}\right) a_{(2)}=\epsilon(a) \mathbf{1}
$$

Proposition 2.5 The antipode in a Hopf algebra $H$ is an anti-algebra and anti-coalgebra homomorphism, that is,

$$
S(a b)=S(b) S(a), \quad \Delta(S(a))=\sum S\left(a_{(2)}\right) \otimes S\left(a_{(1)}\right), \quad \forall a, b \in H .
$$

Proof. Let $a, b$ be two arbitrary elements of the Hopf algebra $H$,

$$
\begin{aligned}
S(a b) & =\sum \epsilon\left(b_{(1)}\right) S\left(a b_{(2)}\right)=\sum S\left(b_{(1)}\right) b_{(2)} S\left(a b_{(3)}\right)= \\
& =\sum S\left(b_{(1)}\right) \epsilon\left(a_{(1)}\right) b_{(2)} S\left(a_{(2)} b_{(3)}\right)= \\
& =\sum S\left(b_{(1)}\right) S\left(a_{(1)}\right) a_{(2)} b_{(2)} S\left(a_{(3)} b_{(3)}\right)= \\
& =\sum S\left(b_{(1)}\right) S\left(a_{(1)}\right)(a b)_{(2)} S\left((a b)_{(3)}\right)=\sum S\left(b_{(1)}\right) S\left(a_{(1)}\right) \epsilon\left((a b)_{(2)}\right) \\
& =\sum S\left(b_{(1)} \epsilon\left(b_{(2)}\right)\right) S\left(a_{(1)} \epsilon\left(a_{(2)}\right)\right)=S(b) S(a) .
\end{aligned}
$$

In the first and third equalities, we use the axiom of co-unit, in the second and fourth equalities, we use the axiom of antipode. The fifth equality is valid because the co-product is a homomorphism of algebra and the sixth again because of the axiom of antipode. Finally, in the seventh equality we use the fact that the co-unit is also an algebra homomorphism and in the last equality we use again the axiom of the co-unit. 
Take now an arbitrary element $a \in H$,

$$
\begin{aligned}
\Delta(S(a)) & =(\mathbf{1} \otimes \mathbf{1}) \Delta(S(a))=\sum\left(\mathbf{1} \otimes \mathbf{1} \epsilon\left(a_{(1)}\right)\right) \Delta\left(S\left(a_{(2)}\right)\right)= \\
& =\sum\left(\mathbf{1} \otimes S\left(a_{(1)}\right) a_{(2)}\right) \Delta\left(S\left(a_{(3)}\right)\right)= \\
& =\sum\left(\mathbf{1} \epsilon\left(a_{(2)}\right) \otimes S\left(a_{(1)}\right) a_{(3)}\right) \Delta\left(S\left(a_{(4)}\right)\right)= \\
& =\sum\left(S\left(a_{(2)}\right) a_{(3)} \otimes S\left(a_{(1)}\right) a_{(4)}\right) \Delta\left(S\left(a_{(5)}\right)\right)= \\
& =\sum\left(S\left(a_{(2)}\right) \otimes S\left(a_{(1)}\right)\right)\left(a_{(3)} \otimes a_{(4)}\right) \Delta\left(S\left(a_{(5)}\right)\right)= \\
& =\sum\left(S\left(a_{(2)}\right) \otimes S\left(a_{(1)}\right)\right) \Delta\left(a_{(3)}\right) \Delta\left(S\left(a_{(4)}\right)\right)= \\
& =\sum\left(S\left(a_{(2)}\right) \otimes S\left(a_{(1)}\right)\right) \Delta\left(a_{(3)} S\left(a_{(4)}\right)\right)= \\
& =\sum\left(S\left(a_{(2)}\right) \otimes S\left(a_{(1)}\right)\right)\left(\mathbf{1} \epsilon\left(a_{(3)}\right) \otimes \mathbf{1}\right)= \\
& =\sum S\left(a_{(2)} \epsilon\left(a_{(3)}\right)\right) \otimes S\left(a_{(1)}\right)= \\
& =\sum S\left(a_{(2)}\right) \otimes S\left(a_{(1)}\right) .
\end{aligned}
$$

The second and fourth equalities are instances of the co-unit axiom, while the third and fifth equalities are instances of the antipode axiom. The sixth equality is merely a factorization of the product in $H \otimes H$. The eighth equality is given by the fact that the co-product is an algebra homomorphism. Finally, we use again the axiom of antipode and the axiom of co-unit to obtain the result.

Example 2.6 The algebra of coordinate functions on the Lie group $S L(n)$ is isomorphic, as algebra, to $\mathbb{C}\left[t_{i j}\right] /\langle\operatorname{det}(t)-1\rangle$, where the indices are taken between 1 and $n$ and $\operatorname{det}(t)$ is the determinant of the matrix with entries $t_{i j}$. The Hopf algebra structure is given by

$$
\Delta\left(t_{i j}\right)=\sum_{k=1}^{n} t_{i k} \otimes t_{k j}, \quad \epsilon\left(t_{i j}\right)=\delta_{i j}, \quad S\left(t_{i j}\right)=\left(t^{-1}\right)_{i j}
$$

where the symbol $\left(t^{-1}\right)_{i j}$ denotes the $i j$-th entry of the inverse matrix $t^{-1}$. This Hopft algebra is commutative, but clearly non co-commutative.

Example 2.7 The universal envelopping algebra of a Lie algebra $\mathfrak{g}$ is the algebra defined by $\mathcal{U}(\mathfrak{g})=T(\mathfrak{g}) / \mathcal{I}$ where $T(\mathfrak{g})$ is the tensor algebra over $\mathfrak{g}$ and $\mathcal{I}$ is 
the ideal generated by elements of the form $X \otimes Y-Y \otimes X-[X, Y], \forall X, Y \in \mathfrak{g}$. The Hopf structure is given by

$$
\Delta(X)=X \otimes \mathbf{1}+\mathbf{1} \otimes X, \quad \epsilon(X)=0, \quad S(X)=-X, \quad \forall X \in \mathfrak{g} .
$$

It is sufficient to define the relations on the elements of $\mathfrak{g}$ because of the universal property of $\mathcal{U}(\mathfrak{g})$. This Hopf algebra is easily verificable to be co-commutative, but not commutative.

Example 2.8 As an important example of both noncommutative and non cocommutative Hopf algebra we have the Hopf algebra $S L_{q}(2)$, generated by elements $a, b, c, d$ and $q$ being a complex number, satisfying the commutation relations $[12]$

$$
\begin{gathered}
a b=q b a, \quad a c=q c a, \quad b d=q d b, \quad c d=q d c, \quad b c=c b \\
a d-d a=\left(q-q^{-1}\right) b c, \quad a d-q b c=1 .
\end{gathered}
$$

The Hopf algebra structure is given by the following relations

$$
\begin{gathered}
\Delta(a)=a \otimes a+b \otimes c, \quad \Delta(b)=a \otimes b+b \otimes d, \\
\Delta(c)=c \otimes a+d \otimes c, \quad \Delta(d)=c \otimes b+d \otimes d, \\
\epsilon(a)=\epsilon(d)=1, \quad \epsilon(b)=\epsilon(c)=0, \\
S(a)=d, \quad S(b)=-q^{-1} b, \quad S(c)=-q c, \quad S(d)=a .
\end{gathered}
$$

It is clear that this Hopf algebra is neither commutative nor co-commutative.

When we put in this Hopf algebra an involution, that is, an anti-linear map $*: S L_{q}(2) \rightarrow S L_{q}(2)$, such that $\left(x^{*}\right)^{*}=a$ and $(x . y)^{*}=y^{*} \cdot x^{*}, \forall x, y \in S L_{q}(2)$ and satisfying

$$
a^{*}=d, \quad b^{*}=-q c, \quad c^{*}=-q^{-1} b, \quad d^{*}=a,
$$

then this algebra is the quantum group $S U_{q}(2)$, which was the first quantum group to appear in the literature [16]. 
Definition 2.9 Given a Hopf algebra $H$ and a vector space $A$ a left action of $H$ on $A$ is a linear map

$$
\begin{aligned}
\triangleright: H \otimes A & \rightarrow A, \\
h \otimes a & \mapsto h \triangleright a
\end{aligned}
$$

satisfying $\triangleright \circ(I d \otimes \triangleright)=\triangleright \circ(\mu \otimes I d)$, and $\triangleright \circ(\eta \otimes I d)=I d$, or in terms of the elements $g \triangleright(h \triangleright a)=(g h) \triangleright a$ and $\mathbf{1} \triangleright a=a, \forall, g, h \in H, \forall a \in A$. A vector space $A$ with a left action of a Hopf algebra $H$ is said to be a left $H$-module.

If $A$ itself is an algebra and the action satisfies $h \triangleright(a b)=\sum\left(h_{(1)} \triangleright a\right)\left(h_{(2)} \triangleright b\right)$, then $A$ is a left $H$-module algebra.

One can also define the notions of right action $\triangleleft: A \otimes H \rightarrow A$, right $H$ module and right $H$-module algebra.

Example 2.10 A Hopf algebra $H$ can act over itself by left and right regular actions

$$
L_{a}(b)=a \triangleright b=a b, \quad R_{a}(b)=b \triangleleft a=b a,
$$

and by left and right adjoint actions,

$$
a d_{L}(a)(b)=a \triangleright b=\sum a_{(1)} b S\left(a_{(2)}\right), \quad a d_{R}(a)(b)=b \triangleleft a=\sum S\left(a_{(1)}\right) b a_{(2)} .
$$

Proposition 2.11 With the left or right adjoint action, $H$ is respectively a left or right $H$-module algebra.

Proof. This proof is instructive in order to be familiar with the techniques of Hopf algebras. Take, for example, the left adjoint action

$$
a d_{L}(a)(b c)=\sum a_{(1)} b c S\left(a_{(2)}\right) .
$$

Using the co-unit axiom, the expression (1) reads

$$
a d_{L}(a)(b c)=\sum a_{(1)} \epsilon\left(a_{(2)}\right) b c S\left(a_{(3)}\right)=\sum a_{(1)} b \epsilon\left(a_{(2)}\right) c S\left(a_{(3)}\right) .
$$

Finally, with the aid of the axiom of antipode, we have, from (2)

$$
a d_{L}(a)(b c)=\sum a_{(1)} b S\left(a_{(2)}\right) a_{(3)} c S\left(a_{(4)}\right)=\left(a d_{L}\left(a_{(1)}\right)(b)\right)\left(a d_{L}\left(a_{(2)}\right)(c)\right) .
$$


Definition 2.12 Given a Hopf algebra $H$ and a vector space $A$, a left co-action of $H$ on $A$ is a linear map

$$
\begin{aligned}
\Delta_{L}: A & \rightarrow H \otimes A, \\
a & \mapsto \Delta_{L}(a)
\end{aligned}
$$

satisfying the relation $(\Delta \otimes I d) \circ \Delta_{L}=\left(I d \otimes \Delta_{L}\right) \circ \Delta_{L}$, and $(\epsilon \otimes I d) \circ \Delta_{L}=I d$. The vector space $A$ with a left co-action $\Delta_{L}$ of $H$ is said to be an $H$-comodule.

If $A$ itself is an algebra and the co-action satisfies $\Delta_{L}(a b)=\Delta_{L}(a) \Delta_{L}(b)$ and $\Delta_{L}(\mathbf{1})=\mathbf{1} \otimes \mathbf{1}$, the $A$ is a left $H$-comodule algebra.

One can also define right co-actions $\Delta_{R}: A \rightarrow a \otimes H$, right $H$ comodules and right $H$-comodule algebras.

In the Sweedler notation, a left and a right co-action can be represented as

$$
\Delta_{L}(a) \sum a^{(-1)} \otimes a^{(0)} \quad \Delta_{R}(a) \sum a^{(0)} \otimes a^{(1)} .
$$

In this notation, the axioms for co-actions read

$$
\begin{aligned}
\sum a^{(-1)}{ }_{(1)} \otimes a^{(-1)}{ }_{(2)} \otimes a^{(0)} & =\sum a^{(-1)} \otimes a^{(0)(-1)} \otimes a^{(0)(0)} \\
\sum a^{(0)} \otimes a^{(1)}{ }_{(1)} \otimes a^{(1)}{ }_{(2)} & =\sum a^{(0)(0)} \otimes a^{(0)(1)} \otimes a^{(1)} \\
\sum \epsilon\left(a^{(-1)}\right) a^{(0)} & =a \\
\sum a^{(0)} \epsilon\left(a^{(1)}\right) & =a .
\end{aligned}
$$

Example 2.13 A Hopf algebra $H$ co-acts on itself by the co-product and because the co-product is an algebra homomorphism and the co-unit axiom is valid, we can easily show that $H$ is an $H$-comodule algebra (left and right).

Example 2.14 Let $H$ be a Hopf algebra, the left and right adjoint co-actions of $H$ on itself are defined as

$$
A d^{L}(a)=\sum a_{(1)} S\left(a_{(3)}\right) \otimes a_{(2)}, \quad A d^{R}(a)=\sum a_{(2)} \otimes S\left(a_{(1)}\right) a_{(3)} .
$$

More examples can be found in references [13, 12]. 


\section{Covariant Differential Calculus}

In the following considerations, $A$ denotes an arbitrary algebra and $H$ a Hopf algebra. Let $\delta_{L}: A \rightarrow H \otimes A$ denote a left co-action of $H$ on $A$. Similarly, $\delta_{R}: A \rightarrow A \otimes H$ denotes right co-action of $H$ on $A$. The algebra $A$ is to be thought as a left (right) $H$-comodule algebra.

Definition 3.1 A first order differential calculus (FODC) over an algebra $A$ is a pair $(\Gamma, d)$, where $\Gamma$ is an $A$-bimodule and $d: A \rightarrow \Gamma$ is a linear map satisfying the Leibniz rule

$$
d(a b)=a \cdot d b+(d a) \cdot b, \quad \forall a, b \in A
$$

such that $\Gamma=\operatorname{span}\{a \cdot d b \mid a, b \in A\}$. The elements of a FODC $\Gamma$ are called 1-forms.

Two FODC, $\Gamma_{1}$ and $\Gamma_{2}$, over $A$ are isomorphic if there exists a bijective linear map $\phi: \Gamma_{1} \rightarrow \Gamma_{2}$ such that $\phi\left(a \cdot d_{1} b \cdot c\right)=a \cdot d_{2} b \cdot c, \forall a, b, c \in A$.

Example 3.2 Consider $A=\mathbb{C}[x]$, the polynomial algebra over $\mathbb{C}$ in one variable. Let $\Gamma$ be the free left $A$-module generated by the generic element $\mathrm{d} x$. Fixing a polynomial $p \in A$, we can define the right $A$-module structure by $\mathrm{d} x \cdot x=p(x) \mathrm{d} x$. One can prove that for each $p$ we have a unique $A$-bimodule structure on $\Gamma$. For the derivation $\mathrm{d}: A \rightarrow \Gamma$ we define recursively

$$
\mathrm{d}(x)=\mathrm{d} x, \quad \mathrm{~d}\left(x^{n}\right)=\sum_{i+j=n-1} x^{i} p(x)^{j} \mathrm{~d} x,
$$

and extend linearly to $A$.

Let us consider some particular examples. For $p(x)=x$, we have the ordinary commutative differential calculus in one variable. If $p(x)=q x, q \neq 1$ then we have

$$
\mathrm{d}\left(x^{n}\right)=\sum_{i+j=n-1} x^{i} q^{j} x^{j} \mathrm{~d} x=\sum_{j=0}^{n-1} q^{j} x^{n-1} d x=\frac{q^{n}-1}{q-1} x^{n-1} \mathrm{~d} x .
$$

In this case we have the following general expresion:

$$
\mathrm{d} f(x)=D_{q} f(x) \mathrm{d} x=\frac{f(q x)-f(x)}{x(q-1)} \mathrm{d} x .
$$


The symbol $D_{q} f$ is called the q-derivative of the function $f$. Finally, for $p(x)=$ $x+c$ we have

$$
\mathrm{d}\left(x^{n}\right)=\sum_{i+j=n-1} x^{i}(x+c)^{j} \mathrm{~d} x=\frac{(x+c)^{n}-x^{n}}{c} \mathrm{~d} x .
$$

with general expression

$$
\mathrm{d} f(x)=\frac{f(x+c)-f(x)}{c} \mathrm{~d} x .
$$

Definition 3.3 Let $A$ be a left (right) $H$-comodule algebra. A FODC over $A$ is said to be left (right) covariant if $\Gamma$ is a left (right) $H$-comodule with left (right) action $\Delta_{L}: \Gamma \rightarrow H \otimes \Gamma\left(\Delta_{R}: \Gamma \rightarrow \Gamma \otimes H\right)$ such that

$$
\Delta_{L}(a d b)=\delta_{L}(a)(I d \otimes d) \delta_{L}(b) .
$$

Respectively

$$
\Delta_{R}(a d b)=\delta_{R}(a)(d \otimes I d) \delta_{R}(b) .
$$

If $A$ is an $H$-bicomodule algebra, then we can define $\Gamma$ as bicovariant if it is both left and right covariant and $\left(\Delta_{L} \otimes I d\right) \circ \Delta_{R}=\left(I d \otimes \Delta_{R}\right) \circ \Delta_{L}$.

Example 3.4 The algebra $A=\mathbb{C}[x]$ of the preceeding example can be considered as the universal envelopping algebra $\mathcal{U}(\mathbb{R})$, with co-product $\Delta(x)=$ $1 \otimes x+x \otimes 1$. The co-action of $A$ on itself can be interpreted as the action of the additive group $\mathbb{R}$ on itself by translations. By an easy calculation, we can verify that the commutative calculus on $A$ given by the polynomial $p(x)=x$ is bicovariant with

$$
\Delta_{L}(\mathrm{~d} x)=1 \otimes \mathrm{d} x
$$

moreover, this is the only bicovariant calculus on $A$, with this co-action.

Going beyond first order calculus, it is possible to construct noncommutative analogues of the De Rham complex of a $\mathcal{C}^{\infty}$ manifold with the property of covariance. 
Definition 3.5 A differential calculus (abbreviated, a DC) over an algebra $A$ is a graded algebra $\Gamma^{\wedge}=\bigoplus_{n=0}^{\infty} \Gamma^{\wedge n}$, that is, a direct sum of vector spaces $\Gamma^{\wedge n}$, with a product $\wedge: \Gamma^{\wedge n} \times \Gamma^{\wedge m} \rightarrow \Gamma^{\wedge(n+m)}$, and a linear map $d: \Gamma^{\wedge} \rightarrow \Gamma^{\wedge}$ of degree one (that is, $\left.d\left(\Gamma^{\wedge n}\right) \subseteq \Gamma^{\wedge(n+1)}\right)$, such that:

1. $d^{2}=0$.

2. $d(\rho \wedge \eta)=d \rho \wedge \eta+(-1)^{n} \rho \wedge d \eta$ for $\rho \in \Gamma^{\wedge n}$ and $\eta \in \Gamma^{\wedge}$.

3. $\Gamma^{\wedge 0}=A$ and $\Gamma^{\wedge n}=\operatorname{span}\left\{a_{0} d a_{1} \wedge \ldots \wedge d a_{n} \mid a_{0}, \ldots, a_{n} \in A\right\}$.

The elements of each subspace $\Gamma^{n}$ are called $n$-forms.

Proposition 3.6 Let $\Gamma^{\wedge}$ be a DC over the algebra $A$, then we have the following:

a) The pair $\left(\Gamma^{\wedge 1}, d_{\mid \Gamma^{\wedge 0}}\right)$ is a FODC over $A$.

b) $d\left(a_{0} d a_{1} \wedge \ldots \wedge d a_{n}\right)=d a_{0} \wedge d a_{1} \wedge \ldots \wedge d a_{n}$.

c) $\left(a_{0} d a_{1} \wedge \ldots \wedge d a_{n}\right) \wedge\left(a_{n+1} d a_{n+2} \wedge \ldots \wedge d a_{n+k}\right)=(-1)^{n} a_{0} a_{1} d a_{2} \wedge \ldots \wedge$ $d a_{n+k}+\sum_{r=1}^{n}(-1)^{n-r} a_{0} d a_{1} \wedge \ldots \wedge d\left(a_{r} a_{r+1}\right) \wedge \ldots \wedge d a_{n+k}$.

Proof. (a) The item 3) in the definition of a DC over $A$ assures that $\Gamma^{\wedge 1}=$ $\operatorname{span}\{a \mathrm{~d} b \mid a, b \in A\}$. If $\rho \in A$ and $\eta \in \Gamma^{\wedge}$ then $\rho \wedge \eta=\rho \eta$, then the item 2) in the definition aplplied to $\mathrm{d}_{\mid \Gamma^{\wedge 0}}: A \rightarrow \Gamma^{\wedge 1}$, reduces to the ordinary Leibniz rule, proving that $\Gamma^{\wedge 1}$ is a FODC over $A$.

(b) It is a direct consequence of items 1) and 2) of the definition of DC.

(c) This statement is proved by induction on $n$ :

For $n=0$ it is trivial and for $n=1$ we have, by the Leibniz rule,

$a_{0} \mathrm{~d} a_{1} \wedge\left(a_{2} \mathrm{~d} a_{3} \wedge \ldots \wedge \mathrm{d} a_{1+k}\right)=-a_{0} a_{1} \mathrm{~d} a_{2} \wedge \ldots \wedge \mathrm{d} a_{1+k}+a_{0} \mathrm{~d}\left(a_{1} a_{2}\right) \wedge \ldots \wedge \mathrm{d} a_{1+k}$

Suppose valid for $n$ and evaluate

$$
\omega=\left(a_{0} \mathrm{~d} a_{1} \wedge \ldots \wedge \mathrm{d} a_{n+1}\right) \wedge\left(a_{n+2} \mathrm{~d} a_{n+3} \wedge \ldots \wedge \mathrm{d} a_{n+1+k}\right),
$$


then we have

$$
\begin{aligned}
\omega= & \left(a_{0} \mathrm{~d} a_{1}\right) \wedge\left(\mathrm{d} a_{2} \wedge \ldots \wedge \mathrm{d} a_{n+1}\right) \wedge\left(a_{n+2} \mathrm{~d} a_{n+3} \wedge \ldots \wedge \mathrm{d} a_{n+1+k}\right)= \\
= & \left(a_{0} \mathrm{~d} a_{1}\right) \wedge\left((-1)^{n} a_{2} \mathrm{~d} a_{3} \wedge \ldots \wedge \mathrm{d} a_{n+1+k}+\right. \\
& \left.+\sum_{r=1}^{n}(-1)^{n-r} \mathrm{~d} a_{2} \wedge \ldots \wedge \mathrm{d}\left(a_{r+1} a_{r+2}\right) \wedge \ldots \wedge \mathrm{d} a_{n+1+k}\right)= \\
= & (-1)^{n+1} a_{0} a_{1} \mathrm{~d} a_{2} \wedge \ldots \wedge \mathrm{d} a_{n+k}+ \\
& +\sum_{r=1}^{n+1}(-1)^{n+1-r} a_{0} \mathrm{~d} a_{1} \wedge \ldots \wedge \mathrm{d}\left(a_{r} a_{r+1}\right) \wedge \ldots \wedge \mathrm{d} a_{n+1+k}
\end{aligned}
$$

This previous result shows that the basic properties of a differential complex are preserved in a noncommutative context. In order to construct noncommutative differential calculi, let us present a DC over an algebra $A$ which is universal, in the sense that every other DC over $A$ can be obtained by a suitable quotient of this calculus.

Example 3.7 Let $A$ be an algebra and let $\Omega^{1}(A)=A^{2} \subseteq A \otimes A$ be the kernel of the multiplication in $A$. We define also $\mathrm{d}: A \rightarrow A^{2}$ by $\mathrm{d} a=\mathbf{1} \otimes a-a \otimes \mathbf{1}$. It is easy to see that $d$ satisfies the Leibniz rule:

$$
\begin{aligned}
\mathrm{d}(a b) & =\mathbf{1} \otimes a b-a b \otimes \mathbf{1}=\mathbf{1} \otimes a b-a \otimes b+a \otimes b-a b \otimes \mathbf{1}= \\
& =(\mathrm{d} a) b+a \mathrm{~d} b .
\end{aligned}
$$

It is obvious that $a \mathrm{~d} b \in A^{2}$ for all $a, b \in A$, now let $a \otimes b \in A^{2}$, we can write

$$
a \otimes b=a \otimes b-a b \otimes \mathbf{1}=a \mathrm{~d} b .
$$

Therefore, $\Omega^{1}(A)$ is a FODC.

Now, define the $A$ bimodules $\Omega^{n}=\Omega^{1}(A) \otimes_{A} \ldots \otimes_{A} \Omega^{1}(A)$ (with $n$ factors $\left.\Omega^{1}(A)\right)$ and take the tensor algebra $\Omega(A)=\bigoplus_{n=0}^{\infty} \Omega^{n}(A)$, where $\Omega^{0}(A)=A$ and the product is given by the tensor product over $A$. The balanced character of the tensor product over $A$ and the Leibniz rule in each tensor factor allows one to write

$$
\Omega^{n}(A)=\operatorname{span}\left\{a_{0} \mathrm{~d} a_{1} \otimes_{A} \mathrm{~d} a_{2} \otimes_{A} \ldots \otimes_{A} \mathrm{~d} a_{n} \mid a_{0}, a_{1}, \ldots, a_{n} \in A\right\}
$$


Finally, if $\mathrm{d}: \Omega(A) \rightarrow \Omega(A)$ is a linear map such that on monomials is given by

$$
\mathrm{d}\left(a_{0} \mathrm{~d} a_{1} \otimes_{A} \mathrm{~d} a_{2} \otimes_{A} \ldots \otimes_{A} \mathrm{~d} a_{n}\right)=\mathrm{d} a_{0} \otimes_{A} \mathrm{~d} a_{1} \otimes_{A} \mathrm{~d} a_{2} \otimes_{A} \ldots \otimes_{A} \mathrm{~d} a_{n}
$$

then $\mathrm{d}^{2}=0$ and this makes $\Omega(A)$ a DC over $A$, namely, the differential envelope of $A$.

The universal character of $\Omega(A)$ can be easily shown: Given another DC over $A,\left(\Gamma^{\wedge}, \widetilde{\mathrm{d}}\right)$, define a linear map

$$
\begin{aligned}
& \psi: \quad \Omega(A) \quad \rightarrow \Gamma^{\wedge} . \\
& a_{0} \mathrm{~d} a_{1} \otimes_{A} \ldots \otimes_{A} \mathrm{~d} a_{n} \mapsto a_{0} \tilde{\mathrm{d}} a_{1} \wedge \ldots \wedge \widetilde{\mathrm{d}} a_{n}
\end{aligned}
$$

The universal character of the tensor algebra extends automatically the linear map $\psi$ to a unique algebra homomorphism. By construction, the map $\psi$ is surjective and one can verify trivially that $\psi \circ \mathrm{d}=\widetilde{\mathrm{d}} \circ \psi$. The $\operatorname{subspace} \mathcal{N}=\operatorname{Ker} \psi$ is in fact a bilateral ideal of $\Omega(A)$ and because $\psi$ intertwins the differentials $\mathrm{d}$ and $\widetilde{\mathrm{d}}$ we can prove that $\mathrm{d}(\mathcal{N}) \subseteq \mathcal{N}$. Therefore, we have the isomorphism $\Gamma^{\wedge} \cong$ $\Omega(A) / \mathcal{N}$. The FODC $\Omega^{1}(A)$ is a universal FODC, considering the restriction of the above isomorphism.

Definition 3.8 Let $A$ be a left (right) $H$-comodule algebra and $\Gamma^{\wedge}$ a DC over A. The calculus $\Gamma^{\wedge}$ is said to be left (right) covariant if there exists an algebra homomorphism $\Delta_{L}: \Gamma^{\wedge} \rightarrow H \otimes \Gamma^{\wedge}$ (resp. $\Delta_{R}: \Gamma^{\wedge} \rightarrow \Gamma^{\wedge} \otimes H$ ), such that

$$
\begin{aligned}
& \Delta_{L}\left(a_{0} d a_{1} \wedge \ldots \wedge d a_{n}\right)=\sum a_{0}^{(-1)} a_{1}^{(-1)} \ldots a_{n}^{(-1)} \otimes a_{0}^{(0)} d a_{1}^{(0)} \wedge \ldots \wedge d a_{n}^{(0)} \\
& \Delta_{R}\left(a_{0} d a_{1} \wedge \ldots \wedge d a_{n}\right)=\sum a_{0}^{(0)} d a_{1}^{(0)} \wedge \ldots \wedge d a_{n}^{(0)} \otimes a_{0}^{(1)} a_{1}^{(1)} \ldots a_{n}^{(1)} .
\end{aligned}
$$

Proposition 3.9 If $A$ is a left (right) $H$-comodule algebra, then the universal $F O D C \Omega^{1}(A)$ and the universal $D C \Omega(A)$ is left (right) covariant.

Proof. Given two left $H$-comodules $A$ and $B$, one can define a left $H$-comodule structure on $A \otimes B$ by

$$
\Delta_{L}(a \otimes b)=\sum a^{(-1)} b^{(-1)} \otimes a^{(0)} \otimes b^{(0)}
$$

The right $H$-comodule structure is given in a similar manner. 
Take an element $a \mathrm{~d} b \in \Omega^{1}(A)$, then

$$
\begin{aligned}
\Delta_{L}(a \mathrm{~d} b) & =\Delta_{L}(a \otimes b-a b \otimes \mathbf{1})= \\
& =\sum a^{(-1)} b^{(-1)} \otimes a^{(0)} \otimes b^{(0)}-\sum a^{(-1)} b^{(-1)} \otimes a^{(0)} b^{(0)} \otimes \mathbf{1}= \\
& =\sum a^{(-1)} b^{(-1)} \otimes a^{(0)} \mathrm{d} b^{(0)}=\delta_{L}(a)(\mathrm{Id} \otimes \mathrm{d}) \delta_{L}(b) .
\end{aligned}
$$

This makes $\Omega^{1}(A)$ a left covariant FODC. The right covariant structure can be constructed similarly.

Using the universal property of the tensor algebra, we extend $\Delta_{L}$ uniquely as an algebra homomorphism on $\Omega(A)$ and verify that this extension turns $\Omega(A)$ into a left covariant DC over $A$. The same for the right covariance.

One important class of examples of covariant differential calculi is given in the case when the algebra $A$ is a Hopf algebra and the covariance is considered with respect to the co-action of $A$ on itself by the co-product. In this case, there exists another characterization of the universal FODC, as shown in the following theorem:

Theorem 3.10 Let $A$ be a Hopf algebra. Then the universal left covariant FODC $\Omega^{1}(A)$ is isomorphic to $A \otimes \operatorname{Ker\epsilon }=A \omega(A)$, where

$$
\begin{aligned}
\omega: \quad A & \rightarrow A \otimes \text { Kerє } \\
a & \mapsto \mathbf{1} \otimes(a-\epsilon(a) \mathbf{1})
\end{aligned}
$$

The correspondence is given by the identifications $\omega(a)=\sum S\left(a_{(1)}\right) d a_{(2)}$ and $d a=\sum a_{(1)} \omega\left(a_{(2)}\right), \forall a \in A$. Moreover, the one forms $\omega(a)$ are left invariant, that is, $\Delta_{L}(\omega(a))=\mathbf{1} \otimes \omega(a), \forall a \in A$.

Proof. Define the following two linear transformations:

$$
\begin{aligned}
\Psi: A^{2} & \rightarrow A \otimes \operatorname{Ker} \epsilon \\
a \otimes b & \mapsto \sum a b_{(1)} \otimes\left(b_{(2)}-\epsilon\left(b_{(2)}\right) \mathbf{1}\right) \\
\Phi: \quad A \otimes \operatorname{Ker} \epsilon & \rightarrow A^{2} . \\
a \otimes b & \mapsto \sum a S\left(b_{(1)}\right) \otimes b_{(2)}
\end{aligned}
$$


Let us verify that these linear maps are mutually inverses. Take an arbitrary element $a \otimes b \in A^{2}$,

$$
\begin{aligned}
\Phi(\Psi(a \otimes b)) & =\sum \Phi\left(a b_{(1)} \otimes\left(b_{(2)}-\epsilon\left(b_{(2)}\right) \mathbf{1}\right)\right)= \\
& =\sum \Phi\left(a b_{(1)} \otimes b_{(2)}\right)-\Phi(a b \otimes \mathbf{1})= \\
& =\sum a b_{(1)} S\left(b_{(2)}\right) \otimes b_{(3)}=a \otimes \sum \epsilon\left(b_{(1)}\right) b_{(2)}=a \otimes b
\end{aligned}
$$

The second equality is due to the co-unit axiom, the third equality is due to the fact that $a b=0$, the fourth equality is due to the antipode axiom and finally the fifth equality again due to the co-unit axiom.

Now take an arbitrary element $a \otimes b \in A \otimes \operatorname{Ker} \epsilon$,

$$
\begin{aligned}
\Psi(\Phi(a \otimes b)) & =\sum \Psi\left(a S\left(b_{(1)}\right) \otimes b_{(2)}\right)=\sum a S\left(b_{(1)}\right) b_{(2)} \otimes\left(b_{(3)}-\epsilon\left(b_{(3)}\right) \mathbf{1}\right)= \\
& =a \otimes \sum \epsilon\left(b_{(1)}\right) b_{(2)}-a \otimes \epsilon\left(\sum \epsilon\left(b_{(1)}\right) b_{(2)}\right) \mathbf{1}= \\
& =a \otimes b-a \otimes \epsilon(b) \mathbf{1}=a \otimes b .
\end{aligned}
$$

The third equality is given by the antipode axiom and by the linearity of the co-unit, the fourth equality by the co-unit axiom and the last equality by the fact that $\epsilon(b)=0$.

With these two isomorphisms, we can identify the one form $\mathrm{d} a=\mathbf{1} \otimes a-a \otimes \mathbf{1}$ with the corresponding one form $\sum a_{(1)} \otimes\left(a_{(2)}-\epsilon\left(a_{(2)}\right) \mathbf{1}\right)=\sum a_{(1)} \omega\left(a_{(2)}\right)$, and in the same manner, identify $\omega(a)=\mathbf{1} \otimes(a-\epsilon(a) \mathbf{1})$ with the corresponding one form $\sum S\left(a_{(1)}\right) \mathrm{d} a_{(2)}$.

Let us verify the invariance of $\omega$. Remembering that the co-action of $A$ on itself is given by the co-product, we have

$$
\Delta_{L}(a \mathrm{~d} b)=\Delta(a)(\mathrm{Id} \otimes \mathrm{d}) \Delta(b), \quad \forall a, b \in A .
$$

Take an arbitrary element $a \in A$,

$$
\Delta_{L}(\omega(a))=\Delta_{L}\left(\sum S\left(a_{(1)}\right) \mathrm{d} a_{(2)}\right)=
$$




$$
\begin{aligned}
& =\sum\left(S\left(a_{(2)}\right) \otimes S\left(a_{(1)}\right)\right)(\mathrm{Id} \otimes \mathrm{d})\left(a_{(3)} \otimes a_{(4)}\right)= \\
& =\sum S\left(a_{(2)}\right) a_{(3)} \otimes S\left(a_{(1)}\right) \mathrm{d} a_{(4)}=\mathbf{1} \otimes S\left(a_{(1)} \epsilon\left(a_{(2)}\right)\right) \mathrm{d} a_{(3)}= \\
& =\mathbf{1} \otimes S\left(a_{(1)}\right) \mathrm{d} a_{(2)}=\mathbf{1} \otimes \omega(a)
\end{aligned}
$$

Note that the concept of left invariant differential one form can be constructed on any left covariant FODC $(\Gamma, \mathrm{d})$ over $A$. The expression is the same, $\omega(a)=\sum S\left(a_{(1)}\right) \mathrm{d} a_{(2)}$. Because of that expression, it is easy to note that $\omega(\mathbf{1})=0$. In what follows, we will write $\omega_{U}$ to denote left invariant one forms in the universal FODC $\Omega^{1}(A)$ and $\omega$ to denote left invariant one forms in any FODC.

Definition 3.11 A bicovariant FODC over a Hopf algebra $A$ is a FODC which is at the same time left and right covariant and

$$
\left(I d \otimes \Delta_{R}\right) \circ \Delta_{L}=\left(\Delta_{L} \otimes I d\right) \circ \Delta_{R} .
$$

The left invariant one forms are not necessarily right invariant, nevertheless, we have the following result:

Proposition 3.12 Let $\Gamma$ be a bicovariant FODC over a Hopf algebra $A$ and let $\omega(a)=\sum S\left(a_{(1)}\right) d a_{(2)}$ be the left invariant one forms in $\Gamma$. Then $\Delta_{R} \circ \omega=$ $(\omega \otimes I d) \circ A d^{R}$.

Proof. Take an arbitrary element $a \in A$,

$$
\begin{aligned}
\Delta_{R}(\omega(a)) & =\Delta_{R}\left(\sum S\left(a_{(1)}\right) \mathrm{d} a_{(2)}\right)= \\
& =\sum\left(S\left(a_{(2)}\right) \otimes S\left(a_{(1)}\right)\right)(\mathrm{d} \otimes \mathrm{Id})\left(a_{(3)} \otimes a_{(4)}\right)= \\
& =\sum S\left(a_{(2)}\right) \mathrm{d} a_{(3)} \otimes S\left(a_{(1)}\right) a_{(4)}= \\
& =\sum \omega\left(a_{(2)}\right) \otimes S\left(a_{(1)}\right) a_{(3)}
\end{aligned}
$$

The following result relates the bicovariant first order differential with $A d^{R}$ invariant right ideals of $\operatorname{Ker} \epsilon \subseteq A$, that is, ideals $\mathcal{R} \subseteq \operatorname{Ker} \epsilon$ such that $A d^{R}(\mathcal{R}) \subseteq$ 
$\mathcal{R} \otimes A$. This result is one of the most important properties of bicovariant differential calculi and provides a full characterization of them only in terms of the structure of ideals of the underlying Hopf algebra.

Theorem 3.13 (i) Let $\mathcal{R}$ be an $A d^{R}$ invariant right ideal of Kere $\subseteq A$, then $\mathcal{N}=A \omega_{U}(\mathcal{R})$ is an $A$-sub bimodule of $\Omega^{1}(A)$ and the quotient $\Gamma=\Omega^{1}(A) / \mathcal{N}$ is a bicovariant FODC over $A$ and $\mathcal{R}=\{a \in \operatorname{Ker\epsilon } \mid \omega(a)=0\}$.

(ii) Conversely, if $\Gamma$ is a bicovariant FODC, then $\mathcal{R}_{\Gamma}=\{a \in \operatorname{Ker\epsilon } \mid \omega(a)=0\}$ is an $A d^{R}$ invariant right ideal of Kere and $\Gamma=\Omega^{1}(A) / A \omega_{U}\left(\mathcal{R}_{\Gamma}\right)$.

Proof. (i) First, let us take a closer look on the $A$-bimodule structure of $\Omega^{1}(A)=A \omega_{U}(A)$ :

$$
\begin{aligned}
a \omega_{U}(b) \cdot c & =\sum a S\left(b_{(1)}\right)\left(\mathrm{d} b_{(2)}\right) c= \\
& =\sum a S\left(b_{(1)}\right) \mathrm{d}\left(b_{(2)} c\right)-\sum a S\left(b_{(1)}\right) b_{(2)} \mathrm{d} c= \\
& =\sum a S\left(b_{(1)}\right) b_{(2)} c_{(1)} \omega_{U}\left(b_{(3)} c_{(2)}\right)-\sum a \epsilon(b) c_{(1)} \omega_{U}\left(c_{(2)}\right)= \\
& =\sum a c_{(1)} \omega_{U}\left(\epsilon\left(b_{(1)}\right) b_{(2)} c_{(2)}\right)-\sum a c_{(1)} \omega_{U}\left(\epsilon(b) c_{(2)}\right)= \\
& =\sum a c_{(1)} \omega_{U}\left((b-\epsilon(b) \mathbf{1}) c_{(2)}\right)= \\
& =\sum a c_{(1)} \omega_{U}\left(\bar{b} c_{(2)}\right) .
\end{aligned}
$$

The left $A$-module structure of $\mathcal{N}$ is evident, to verify the right $A$-module structure, take an arbitrary element $a \omega_{U}(b) \in \mathcal{N}$ and $c \in A$,

$$
\begin{aligned}
a \omega_{U}(b) \cdot c & =\sum a c_{(1)} \omega_{U}\left(b c_{(2)}\right)= \\
& =\sum a c_{(1)} \omega_{U}\left(b\left(c_{(2)}-\epsilon\left(c_{(2)}\right) \mathbf{1}\right)\right)+a \sum c_{(1)} \epsilon\left(c_{(2)}\right) \omega_{U}(b)= \\
& =\sum a c_{(1)} \omega_{U}\left(b \bar{c}_{(2)}\right)+a c \omega_{U}(b) \in \mathcal{N},
\end{aligned}
$$

the first term is in $\mathcal{N}$ because $b \in \mathcal{R}$ and $\mathcal{R}$ is a right ideal of $\operatorname{Ker} \epsilon$, the second term is evidently in $\mathcal{N}$. Then $\mathcal{N}$ is a sub-bimodule of $\Omega^{1}(A)$ and $\Gamma=\Omega^{1}(A) / \mathcal{N}$ is a FODC.

The covariance is verified if one can prove that $\Delta_{L}(\mathcal{N}) \subseteq A \otimes \mathcal{N}$, and $\Delta_{R}(\mathcal{N}) \subseteq \mathcal{N} \otimes A$

$$
\Delta_{L}\left(a \omega_{U}(b)\right)=\Delta(a)\left(\mathbf{1} \otimes \omega_{U}(b)\right)=\sum a_{(1)} \otimes a_{(2)} \omega_{U}(b) .
$$


Similarly

$$
\Delta_{R}\left(a \omega_{U}(b)\right)=\Delta(a)(\omega \otimes \operatorname{Id}) A d^{R}(b)
$$

which is in $\mathcal{N}$ because, by hypothesis $\mathcal{R}$ is $A d^{R}$ invariant. Therefore, $\Gamma$ is a bicovariant FODC.

It remains to verify that $\mathcal{R}=\{a \in \operatorname{Ker} \epsilon \mid \omega(a)=0\}=\mathcal{R}_{\Gamma}$. If $a \in \mathcal{R}$, then

$$
\begin{aligned}
\omega(a) & =\sum S\left(a_{(1)}\right) \mathrm{d} a_{(2)}=\sum S\left(a_{(1)}\right)\left(\mathbf{1} \otimes a_{(2)}-a_{(2)} \otimes \mathbf{1}\right)+\mathcal{N}= \\
& =\sum S\left(a_{(1)}\right) a_{(2)} \omega_{U}\left(a_{(3)}\right)+\mathcal{N}=\omega_{U}(a)+\mathcal{N}=\mathcal{N}=0 .
\end{aligned}
$$

Conversely, if $\omega(a)=0$, for some $a \in \operatorname{Ker} \epsilon$, then $\omega_{U}(a) \in \mathcal{N}$. It is easy to see that $\omega_{U}(a)=\mathbf{1} \otimes a$ and then we can conclude that $a \in \mathcal{R}$.

(ii) Let $\Gamma$ be a bicovariant FODC, $a \in \mathcal{R}_{\Gamma}$ and $b \in \operatorname{Ker} \epsilon$,

$$
\begin{aligned}
\omega(a b) & =\sum S\left(a_{(1)} b_{(1)}\right) \mathrm{d}\left(a_{(2)} b_{(2)}\right)= \\
& =\sum S\left(b_{(1)}\right) S\left(a_{(1)}\right) a_{(2)} \mathrm{d} b_{(2)}+\sum S\left(b_{(1)}\right) S\left(a_{(1)}\right)\left(\mathrm{d} a_{(2)}\right) b_{(2)}= \\
& =\epsilon(a) \sum S\left(b_{(1)}\right) \mathrm{d} b_{(2)}+\sum S\left(b_{(1)}\right) \omega(a) b_{(2)}=0 .
\end{aligned}
$$

From the fact that $(\omega \otimes \mathrm{Id}) A d^{R}(a)=\Delta_{R}(\omega(a))$ we can conclude easily that $\mathcal{R}_{\Gamma}$ is $A d^{R}$ invariant. The second assertion follows from the item (i).

Example 3.14 Any Hopf algebra admits a commutative bicovariant differential calculus, choosing the ideal $(\operatorname{Ker} \epsilon)^{2}$. It is easy to see that $(\operatorname{Ker} \epsilon)^{2}$ is in fact a bilateral ideal of Ker $\epsilon$. It remains to verify th $A d^{R}$ invariance. Let $a, b \in \operatorname{Ker} \epsilon$,

$$
\begin{aligned}
(\epsilon \otimes \mathrm{Id} \otimes \mathrm{Id}) A d^{R}(a \otimes b) & =\sum \epsilon\left(a_{(2)}\right) b_{(2)} \otimes S\left(a_{(1)} b_{(1)}\right) a_{(3)} b_{(3)}= \\
& =\sum b_{(2)} \otimes S\left(b_{(1)}\right) S\left(a_{(1)} \epsilon\left(a_{(2)}\right)\right) a_{(3)} b_{(3)}= \\
& =\sum b_{(2)} \otimes \epsilon(a) S\left(b_{(1)}\right) b_{(3)}=0,
\end{aligned}
$$

similarly, we prove that $(\mathrm{Id} \otimes \epsilon \otimes \mathrm{Id}) A d^{R}(a \otimes b)$ and then, we conclude that $A d^{R}(a b) \in(\operatorname{Ker} \epsilon)^{2} \otimes A$.

Next, we have to check that the bicovariant calculus obtained is in fact commutative, in the sense that the invariant one forms commute with elements 
of $A$. Consider $a, b \in A$,

$$
\begin{aligned}
\omega(b) a & =a_{(1)} \omega\left(\bar{b} a_{(2)}\right)=a_{(1)} \omega\left(\bar{b} \bar{a}_{(2)}\right)+a_{(1)} \omega\left(\bar{b} \epsilon\left(a_{(2)}\right)\right)= \\
& =a \omega(b-\epsilon(b) \mathbf{1})=a \omega(b) .
\end{aligned}
$$

Example 3.15 An interesting class of bicovariant differential calculi can be obtained for universal envelopping algebras $\mathcal{U}(\mathfrak{g})$ [15]. Consider an irreducible right $\mathcal{U}(\mathfrak{g})$-module, $(\mathbb{V}, \rho)$. Take $\Lambda \in \mathbb{V}$ and define $\rho_{\Lambda}: \mathcal{U}(\mathfrak{g}) \rightarrow \mathbb{V}$ as $\rho_{\Lambda}(a)=$ $\Lambda \cdot \rho(a)$. In the universal envelopping algebra $\operatorname{Ker} \epsilon=\mathcal{U}(\mathfrak{g}) \backslash(\mathbb{C} 1)$, and we can easily see that $\operatorname{Ker} \rho_{\Lambda}$ is a right ideal of $\operatorname{Ker} \epsilon$. The $A d^{R}$ invariance follows from the co-commutativity of $\mathcal{U}(\mathfrak{g})$ :

$$
\begin{aligned}
A d^{R}(a) & =\sum a_{(2)} \otimes S\left(a_{(1)}\right) a_{(3)}=\sum a_{(3)} \otimes S\left(a_{(1)}\right) a_{(2)}= \\
& =\sum \epsilon\left(a_{(1)}\right) a_{(2)} \otimes \mathbf{1}=a \otimes \mathbf{1} .
\end{aligned}
$$

Then, the bicovariant FODC over $\mathcal{U}(\mathfrak{g})$ can be written as $\Gamma=\mathcal{U}(\mathfrak{g}) \otimes\left(\operatorname{Ker} \epsilon / \operatorname{Ker} \rho_{\Lambda}\right)$ $=\mathcal{U}(\mathfrak{g}) \otimes \mathbb{V}$, this last identification is possible because $\rho$ is an irreducible representation, then $\rho_{\Lambda}$ is surjective. The invariant one forms can be written as $\omega(a)=\rho_{\Lambda}(a), \forall a \in \mathcal{U}(\mathfrak{g})$.

For elements $X \in \mathfrak{g}$ we have $\mathrm{d} X=\omega(X)=\rho_{\Lambda}(X)$,

$$
\mathrm{d} X=\sum X_{(1)} \omega\left(X_{(2)}\right)=X \omega(\mathbf{1})+\mathbf{1} \omega(X)=\omega(X)
$$

The commutation relations between the invariant one forms and the elements of $\mathcal{U}(\mathfrak{g})$ can be verified as follows: Take $v \in \mathbb{V}$ and $X \in \mathfrak{g}$, the surjectivity of $\rho_{\Lambda}$ implies that there exists an element $a \in \mathcal{U}(\mathfrak{g})$ such that $v=\rho_{\Lambda}(a)=\omega(a)$ (for sake of notation, we are writing only $v$ instead of $\mathbf{1} \otimes v$, therefore, we have

$$
\begin{aligned}
v \cdot X & =\rho_{\Lambda}(a) \cdot X=\sum X_{(1)} \rho_{\Lambda}\left(a X_{(2)}\right)=X \rho_{\Lambda}(a)+\rho_{\Lambda}(a X)= \\
& =X \cdot v+v \rho(X) .
\end{aligned}
$$

This allows one to write recursively, for monomials $X_{1} X_{2} \ldots X_{n} \in \mathcal{U}(\mathfrak{g})$, the following expression

$$
\mathrm{d}\left(X_{1} X_{2} \ldots X_{n}\right)=\sum_{k=0}^{n-1} \sum_{\sigma \in S_{(k, n-k)}} X_{\sigma(1)} \ldots X_{\sigma(k)} \rho_{\Lambda}\left(X_{\sigma(k+1)} \ldots X_{\sigma(n)}\right),
$$


where $S_{(k, n-k)}$ is the subgroup of permutations of $S_{n}$ such that $\sigma(1)<\cdots<\sigma(k)$ and $\sigma(k+1)<\cdot<\sigma(n)$, called $(k, n-k)$ shuffles.

The calculi obtained from irreducible representations are irreducible, in the sense that they cannot be expressed as a direct sum of smaller bicovariant FODCs over $\mathcal{U}(\mathfrak{g})$ [15]. There are other non irreducible calculi over $\mathcal{U}(\mathfrak{g})$ which can be constructed using matricial representation, see for example [2].

The theory of bicovariant differential calculi over Quantum Groups was first established in the reference [17]. There, the FODC and the differential calculus over $S U_{q}(2)$ was constructed. This quantum group has two different four dimensional first order differential calculi. The theory of quantum vector fields and quantum Lie algebras are developed in the same reference.

\section{Quantum Principal Bundles}

As seen in previous sections, the formalism of Hopf algebras is very suitable to incorporate group actions in a noncommutative context. A very natural construction in the quantum group approach for noncommutative geometry is the definition of noncommutative principal bundles $[3,11]$.

Definition 4.1 Let $H$ be a Hopf algebra, $P$ be a right $H$-comodule algebra and let

$$
M=P^{H}=\left\{u \in P \mid \Delta_{R}(u)=u \otimes \mathbf{1}\right\}
$$

be the invariant sub-algebra of $P$. We say that $P=P(M, H)$ is quantum principal bundle with structure quantum group $H$, base $M$ and with universal differential calculus if the following conditions are satisfied:

1. The map $\chi=(\mu \otimes I d) \circ\left(I d \otimes \Delta_{R}\right): P \otimes P \rightarrow P \otimes H$ is surjective (freeness condition).

2. $\operatorname{Ker} \chi=\Gamma_{H o r}=P \Omega^{1}(M) P$ (exactness condition).

The first item in the definition of quantum principal bundle is motivated by the fact that the group action on a classical principal bundle is free, that is the 
map $\alpha: P \times G \rightarrow P \times P$ which associates the pair $(u, g)$ to the pair $(u, u \cdot g)$ is injective. The second condition needs some explanation. In the classical case, smoothness and dimension arguments combined with the freeness of the group action ensures that the quotient is a manifold and the fiber through a point $u \in P$ is homeomorphic to the Lie group $G$. At the infinitesimal level, by dimension arguments, the Lie algebra $\mathfrak{g}$ of $G$ is isomorphic, by the map which generates the fundamental vector fields, to the vertical part of $T_{u} P$, for each $u \in P$. In our case one needs to suppose directly that the image of the fundamental vector fields span all the vertical vector fields. In terms of forms we can say that the horizontal forms span the anihilator of the invariant vector fields.

The concept of quantum principal bundle with universal calculus is related to the more algebraic concept of Hopf-Galois extension

Definition 4.2 We say that a right H-comodule algebra is a Hopf Galois extension of $H$ if the canonical map $\bar{\chi}=(\mu \otimes I d) \circ\left(I d \otimes \Delta_{R}\right): P \otimes_{M} P \rightarrow P \otimes H$ is a isomorphism, where $M=P^{H}$ is the right invariant sub-algebra.

Theorem 4.3 A right $H$-comodule algebra $P$ is a Hopf-Galois extension of $H$ if, and only if $P(M, H)$ is a quantum principal bundle with universal calculus.

Proof. Consider the following diagram:

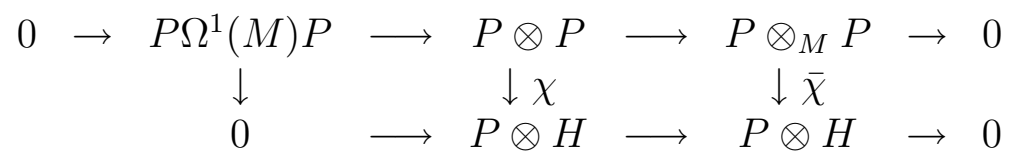

Using the snake lemma, we have the exact sequence

$$
0 \rightarrow P \Omega^{1}(M) P \rightarrow \operatorname{Ker} \chi \rightarrow \operatorname{Ker} \bar{\chi} \rightarrow 0 \rightarrow \text { Coker } \chi \rightarrow \text { Coker } \bar{\chi} \rightarrow 0
$$

Assume first that $P$ is a Hopf-Galois extension, then $\operatorname{Ker} \bar{\chi}=\operatorname{Coker} \bar{\chi}=$ 0. By the exactness of (5), we have Coker $\chi=0$ which express the freeness condition, and we have the isomorphism $P \Omega^{1}(M) P \cong \operatorname{Ker} \chi$, which express the exactness condition. Therefore $P(M, H)$ is a quantum principal bundle with universal calculus. 
On the other hand, if $P(M, H)$ is a quantum principal bundle, then $P \Omega^{1}(M) P$ $\cong \operatorname{Ker} \chi$ and Coker $\chi=0$, again, by the exactness of (5) we have $\operatorname{Ker} \bar{\chi}=0$ and Coker $\bar{\chi}=0$, which implies that $\bar{\chi}$ is an isomorphism and $P$ is a Hopf-Galois extension of $H$.

If the differential calculi involved are not universal, then the definition of quantum principal bundle requires more conditions. Let $\Gamma_{P}$ be the FODC over $P$ and $\mathcal{N}_{P}$ be the sub-bimodule of $\Omega^{1}(P)$ such that $\Gamma_{P}=\Omega^{1}(P) / \mathcal{N}_{P}$. Let $\mathcal{M}_{H} \subseteq$ Ker $\epsilon$ be the right ideal of Ker $\epsilon$ which defines the bicovariant FODC $\Gamma_{H}$. Three conditions are important in order to define the quantum principal bundle: the right $H$ covariance of the FODC $\Gamma_{P}$ and the compatibility of the canonical map $\chi$ and the exactness condition with the sub-bimodules such that the same structure remains valid in the quotient. Let us define first the map $\chi_{\mathcal{N}_{P}}: \Gamma_{P} \rightarrow P \otimes\left(\operatorname{Ker} \epsilon / \mathcal{M}_{H}\right)$ by $\chi_{\mathcal{N}_{P}}(\rho)=\left(\operatorname{Id} \otimes \pi_{H}\right) \circ \chi\left(\rho_{U}\right)$, where $\pi_{H}: \operatorname{Ker} \epsilon \rightarrow$ Ker $\epsilon / \mathcal{M}_{H}$ is the canonical projection and $\rho_{U}$ is a representative in $\pi_{\mathcal{N}_{P}}^{-1}$ where $\pi_{\mathcal{N}_{P}}: \Omega^{1}(P) \rightarrow \Gamma_{P}$ is the respective canonical projection.

Definition 4.4 We say that $P=P\left(M, H, \mathcal{N}_{P}, \mathcal{M}_{H}\right)$ is a quantum principal bundle with structure quantum group $H$ and base $M=P^{H}$ if the following conditions are satisfied:

1. The map $\chi=(\mu \otimes I d) \circ\left(I d \otimes \Delta_{R}\right): P \otimes P \rightarrow P \otimes H$ is surjective (freeness condition).

2. $\Delta_{R}\left(\mathcal{N}_{P}\right) \subseteq \mathcal{N}_{P} \otimes H$ (right covariance of $\left.\Gamma_{P}\right)$.

3. $\chi\left(\mathcal{N}_{P}\right) \subseteq P \otimes \mathcal{M}_{H}$ (fundamental vector fields compatibility condition).

4. $\operatorname{Ker}_{\mathcal{N}_{P}}=\Gamma_{\text {Hor }}$ (exactness condition).

Example 4.5 Let $P$ be a right $H$-comodule algebra with invariant sub-algebra $M=P^{H}$, we say that $P$ is a trivial quantum principal bundle if there exists a linear map $\Phi: H \rightarrow P$ satisfying the following conditions: 
1. $\Phi$ is convolution invertible, that is, there exists a linear map $\bar{\Phi}: H \rightarrow P$ such that $\sum \Phi\left(a_{(1)}\right) \bar{\Phi}\left(a_{(2)}\right)=\sum \bar{\Phi}\left(a_{(1)}\right) \Phi\left(a_{(2)}\right)=\epsilon(a) \mathbf{1}$.

2. $\Phi\left(\mathbf{1}_{H}\right)=\mathbf{1}_{P}$.

3. $\Delta_{R} \circ \Phi=(\Phi \otimes \mathrm{Id}) \circ \Delta$.

From these properties, we can easily conclude that $\Delta_{R} \circ \bar{\Phi}=(\bar{\Phi} \otimes \mathrm{Id}) \circ \sigma \circ \Delta$, where $\sigma$ is the flip morphism in $H \otimes H$.

These bundles are quantum principal bundles with universal calculus, usually known in the Hopf algebra literature as cleft Hopf-Galois extensions. To see this fact, let us first prove that $P$ is isomorphic, as vector space, with $M \otimes H$. As $M \subseteq P$ define a linear map

$$
\begin{aligned}
\Theta: M \otimes H & \rightarrow P, \\
b \otimes a & \mapsto b \Phi(a)
\end{aligned}
$$

whose inverse is given explicitly as $\Theta^{-1}(u)=\sum u^{(0)} \bar{\Phi}\left(u^{(1)}{ }_{(1)}\right) \otimes u^{(1)}{ }_{(2)}$.

Some routine verifications need to be made, for example, that the image of $\Theta^{-1}$ is in fact in $M \otimes H$ :

$$
\begin{aligned}
\left(\Delta_{R} \otimes \mathrm{Id}\right)\left(\Theta^{-1}(u)\right) & \left.=\sum \Delta_{R}\left(u^{(0)}\right)_{R}\left(\bar{\Phi}\left(u^{(1)}{ }_{(1)}\right)\right) \otimes u^{(1)}{ }_{(2)}\right)= \\
& =\sum u^{(0)(0)} \bar{\Phi}\left(u^{(1)}{ }_{(2)}\right) \otimes u^{(0)(1)} S\left(u^{(1)}{ }_{(1)}\right) \otimes u^{(1)}{ }_{(3)}= \\
& =\sum u^{(0)} \bar{\Phi}\left(u^{(1)}{ }_{(2)(2)}\right) \otimes u_{(1)}^{(1)} S\left(u^{(1)}{ }_{(2)(1)}\right) \otimes u^{(1)}{ }_{(2)(3)}= \\
& =\sum u^{(0)} \bar{\Phi}\left(u^{(1)}{ }_{(3)}\right) \otimes u^{(1)}{ }_{(1)} S\left(u^{(1)}{ }_{(2)}\right) \otimes u^{(1)}{ }_{(4)}= \\
& =\sum u^{(0)} \bar{\Phi}\left(u_{(1)}^{(1)}\right) \otimes \mathbf{1} \otimes u_{(2)}^{(1)} .
\end{aligned}
$$

Afterwards, one needs to verify that $\Theta$ and $\Theta^{-1}$ are indeed mutually inverses:

$$
\begin{aligned}
\Theta\left(\Theta^{-1}(u)\right) & =\Theta\left(\sum u^{(0)} \bar{\Phi}\left(u_{(1)}^{(1)}\right) \otimes u_{(2)}^{(1)}\right)= \\
& =\sum u^{(0)} \bar{\Phi}\left(u^{(1)}{ }_{(1)}\right) \Phi\left(u_{(2)}^{(1)}\right)= \\
& =\sum u^{(0)} \epsilon\left(u^{(1)}\right)=u, \\
\Theta^{-1}(\Theta(b \otimes a)) & =\Theta^{-1}(b \Phi(a))= \\
& =b^{(0)} \Phi(a)^{(0)} \bar{\Phi}\left(b^{(1)}{ }_{(1)} \Phi(a)_{(1)}^{(1)}\right) \otimes b_{(2)}^{(1)}{ }_{(a)}{ }_{(2)}^{(1)}= \\
& =\sum b \Phi\left(a_{(1)}\right) \bar{\Phi}\left(a_{(2)}\right) \otimes a_{(3)}= \\
& =b \otimes\left(\sum \epsilon\left(a_{(1)}\right) a_{(2)}\right)=b \otimes a .
\end{aligned}
$$


In order to prove the freeness condition, consider an element $\sum u_{k} \otimes a^{k} \in$ $P \otimes H$ and define $\rho \in \Omega^{1}(P)$ as

$$
\rho=\sum u_{k} \bar{\Phi}\left(a_{(1)}^{k}\right) \otimes \Phi\left(a_{(2)}^{k}\right) .
$$

Then, we have

$$
(\mu \otimes \mathrm{Id}) \circ\left(\mathrm{Id} \otimes \Delta_{R}\right)(\rho)=\sum u_{k} \bar{\Phi}\left(a_{(1)}^{k}\right) \Phi\left(a_{(2)}^{k}\right) \otimes a_{(3)}^{k}=\sum u_{k} \otimes a^{k} .
$$

Finally, it remains to check the exactness condition, that is $\operatorname{Ker} \chi=P \mathrm{~d}(M) P$. The inclusion $\operatorname{Pd}(M) P \subseteq \operatorname{Ker} \chi$ is trivial, take $u, v \in P$ and $b \in M$, then

$$
\begin{aligned}
\chi(u(\mathrm{~d} b) v) & =\chi(u(\mathbf{1} \otimes b-b \otimes \mathbf{1}) v)= \\
& =\chi(u \otimes b v-u b \otimes v)= \\
& =u b^{(0)} v^{(0)} \otimes b^{(1)} v^{(1)}-u b v^{(0)} \otimes v^{(1)}=0 .
\end{aligned}
$$

The inclusion Ker $\chi \subseteq \operatorname{Pd}(M) P$ is less trivial, take in this case an element $\rho=\sum_{i} u_{i} \mathrm{~d} v_{i} \in \operatorname{Ker} \chi$, since $\Phi$ establishes an isomorphism between $P$ and $M \otimes H$, consider $v_{i}=\sum_{k} b_{i}^{k} \Phi\left(a_{i}^{k}\right)$, then we can write

$$
\begin{aligned}
0 & =(\mu \otimes \operatorname{Id}) \circ(\operatorname{Id} \otimes \bar{\Phi} \otimes \Phi) \circ(\operatorname{Id} \otimes \Delta) \circ \chi(\rho)= \\
& =(\mu \otimes \operatorname{Id}) \circ(\operatorname{Id} \otimes \bar{\Phi} \otimes \Phi) \circ(\operatorname{Id} \otimes \Delta)\left(\sum_{i, k} u_{i} b_{i}^{k} \Phi\left(a_{i(1)}^{k}\right) \otimes a_{i(2)}^{k}-u_{i} b_{i}^{k} \Phi\left(a_{i}^{k}\right) \otimes \mathbf{1}\right)= \\
& =(\mu \otimes \operatorname{Id})\left(\sum_{i, k}\left(u_{i} b_{i}^{k} \Phi\left(a_{i(1)}^{k}\right) \otimes \bar{\Phi}\left(a_{i(2)}^{k}\right) \otimes \Phi\left(a_{i(3)}^{k}\right)-u_{i} b_{i}^{k} \Phi\left(a_{i}^{k}\right) \otimes \mathbf{1}\right) \otimes \mathbf{1}\right)= \\
& =\sum_{i, k}\left(u_{i} b_{i}^{k} \otimes \Phi\left(a_{i}^{k}\right)-u_{i} b_{i}^{k} \Phi\left(a_{i}^{k}\right) \otimes \mathbf{1}\right)=\sum_{i, k} u_{i} b_{i}^{k} \mathrm{~d} \Phi\left(a_{i}^{k}\right) .
\end{aligned}
$$

Hence, using the Leibniz rule, we have

$$
\begin{aligned}
\rho & =\sum_{i, k} u_{i} \mathrm{~d}\left(b_{i}^{k} \Phi\left(a_{i}^{k}\right)\right)= \\
& =\sum_{i, k} u_{i}\left(\mathrm{~d} b_{i}^{k}\right) \Phi\left(a_{i}^{k}\right)+\sum_{i, k} u_{i} b_{i}^{k} \mathrm{~d} \Phi\left(a_{i}^{k}\right)= \\
& =\sum_{i, k} u_{i}\left(\mathrm{~d} b_{i}^{k}\right) \Phi\left(a_{i}^{k}\right) \in P(\mathrm{~d} M) P .
\end{aligned}
$$


Example 4.6 An example of nontrivial quantum principal bundle can be given by the quantum Hopf fibration. Here $P=S U_{q}(2)$, with Hopf algebra structure given in the Example 2.8. The structure quantum group is given by the algebra of coordinate functions on $U(1)$, that is the polynomial algebra $\mathbb{C}\left[z, z^{-1}\right]$, where $z^{-1}$ is the inverse of $z$ and with Hopf algebra structure given by

$$
\Delta\left(z^{ \pm 1}\right)=z^{ \pm 1} \otimes z^{ \pm 1}, \quad \epsilon\left(z^{ \pm 1}\right)=1, \quad S\left(z^{ \pm 1}\right)=z^{\mp 1} .
$$

There is a Hopf algebra projection $\pi: S U_{q}(2) \rightarrow U(1)$ defined by $\pi(a)=z$, $\pi(b)=\pi(c)=0$ and $\pi(d)=z^{-1}$. With this projection, we can write the right coaction of $U(1)$ on $S U_{q}(2)$ by $\Delta_{R}=(\mathrm{Id} \otimes \pi) \circ \Delta$. Finally, the quantum two sphere $\mathbb{S}_{q}^{2} \subseteq S U_{q}(2)$ is the invariant sub-algebra. This algebra is generated by $\left\{\mathbf{1}, b_{-}=a b, b_{+}=c d, b_{3}=a d\right\}$ and its relations can be deduced from the relations on $S U_{q}(2)$.

It was shown in the reference [3] that $S U_{q}(2)\left(\mathbb{S}_{q}^{2}, \mathbb{C}\left[z, z^{-1}\right], \pi\right)$ is indeed a nontrivial quantum principal bundle.

The noncommutative geometry from the point of view of quantum groups is greatly developed, and it is possible to construct noncommutative anologues of associated vector bundles, equivariant functions, frame bundles, connections and Riemannian geometry. To the interested reader, more details and important results can be found in references $[3,4,14,15]$.

Acknowledgements. The author would like to thank the organizers of the XIII School of Diferential Geometry, held in São Paulo between July 26th and July 30th, for the opportunity to show to the community of differential geometers some aspects of this exciting branch of Mathematics, the Noncommutative Geometry.

\section{References}

[1] Amelino-Camelia, G., Majid, S., Waves on Noncommutative Spacetime and Gamma-Ray Bursts, Int. J. Mod. Phys. A15 (2000), 4301-4324. 
[2] Batista, E., Majid, S., Noncommutative geometry of angular momentum space $\mathcal{U}(\mathfrak{s u}(2))$, J. Math Phys 44 (2003), 107-137.

[3] Brzezinski, T., Majid, S., Quantum group gauge theory on quantum spaces, Commun. Math. Phys. 157 (1993), 591-638.

[4] Brzezinski, T., Translation Map in Quantum Principal Bundles, hepth/9407145.

[5] Chakraborty, P. S., Pal, A., Spectral Triples and Associated Connesde Rham Complex for the Quantum $S U(2)$ and the Quantum Sphere, mathQA/0210049.

[6] Connes, A., Noncommutative Geometry, Academic Press (1994).

[7] Connes, A., Lott, J., Particle Models and Noncommutative Geometry, Nucl. Phys. B (Proc. Suppl.) 18 (1990), 29-47.

[8] Connes, A., Kreimer, D., Hopf Algebras, Renormalization and Noncommutative Geometry, Commun. Math. Phys. 199 (1998), 203-242.

[9] Drinfel'd, V. G., Quantum Groups, in Proc. Int. Cong. Math. (Berkeley 1986), Am. Math. Soc. (1987).

[10] Gracia-Bondía, J. M., Varilly, J. C., Figueroa, H., Elements of Noncommutative Geometry, Birkhäuser (2001).

[11] Hajac, P., Strong Connections on Quantum Principal Bundles, Commun. Math. Phys. 182 (1996), 579-617.

[12] Klimyk, A., Schmüdgen, K., Quantum Groups and Their Representations, Springer-Verlag (1997).

[13] Majid, S., Foundations of Quantum Group Theory, Cambridge Univ. Press (1995) 
[14] Majid, S., Quantum and Braided Group Riemannian Geometry, J. Geom. Phys. 30 (1999), 113-146.

[15] Majid, S., Riemannian Geometry of Quantum Groups and Finite Groups with Nonuniversal Differentials, Commun. Math. Phys. 225 (2002), 131170.

[16] Woronowicz, S. L., Compact Matrix Pseudogroups,Commun. Math. Phys. 111 (1987), 613-665.

[17] Woronowicz, S. L., Differential Calculus on Compact Matrix Pseudogroups (Quantum Groups), Commun. Math. Phys. 122 (1989), 125-170.

Departamento de Matemática

Universidade Federal de Santa Catarina

88.040-900, Florianópolis-SC, Brazil

E-mail: ebatista@mtm.ufsc.br 